\title{
Inter-organ communication in development and cancer
}

Edel Alvarez-Ochoa ${ }^{1,2, *}$, Francesca Froldi ${ }^{1,2, *}$, Louise Cheng ${ }^{1,2,3}$

1. Peter MacCallum Cancer Centre, 305 Grattan St, Parkville, Victoria, Australia, 3000

2. Sir Peter MacCallum Department of Oncology, University of Melbourne, Parkville, Victoria, Australia, 3010

3. The Department of Anatomy and Neuroscience, University of Melbourne, Parkville, Victoria, Australia 3010

* These authors contributed equally to the manuscript.

Email: louise.cheng@petermac.org

\begin{abstract}
:
Studies in model organisms have demonstrated that extensive communication occurs between distant organs both during development, and in diseases such as cancer. Organs communicate with each other to coordinate growth and reach the correct size, while the fate of tumour cells depend on the outcome of their interaction with the immune system and peripheral tissues. In this review, we outline recent studies in Drosophila, which have enabled an improved understanding of the complex crosstalk between organs in the context of both organismal and tumour growth. We argue that Drosophila is a powerful model organism for studying these interactions, and these studies have the potential for improving our understanding of signalling pathways and candidate factors that mediate this conserved inter-organ crosstalk.
\end{abstract}

\section{Introduction:}

Multicellular organisms coordinate and scale the growth of individual organs with overall body size, and adjust readily to environmental changes during development. This plasticity is mediated by the ability of organs to communicate their relative status in order to coordinate each other's function during nutrient restriction or environmental stress. Upon deregulation of growth control, cells and organs lose their

This is the author manuscript accepted for publication and has undergone full peer review but has not been through the copyediting, typesetting, pagination and proofreading process, which may lead to differences between this version and the Version of Record. Please cite this article as doi: $10.1002 /$ wdev.394

This article is protected by copyright. All rights reserved. 
ability to maintain size and function. In addition to autonomous mechanisms, nonautonomous crosstalk plays a key role in regulating both organ and tumour growth. Many studies have identified novel communication factors and the downstream genetic pathways and outputs of these inter-organ interactions. The growth of a tumour is also shaped by its interaction with the immune system, which can be tumour suppressive or tumour promoting depending on the context. Non-mammalian genetic model organisms such as the fruit fly Drosophila melanogaster have the potential to offer novel insights into how organs communicate thanks to their genetic tractability, short generation time, the ability to manipulate multiple organs independently as well as simultaneously, and the possibility to perform large-scale, unbiased forward genetic screens. Flies' organs are functionally analogous to those of mammals and administer the same metabolic functions; furthermore, many of the inter-organ communication factors currently known are conserved. Here, we aim to give an overview of how organs crosstalk with each other to coordinate decisions to commence and cease proliferation in response to environmental cues, in the context of development as well as cancer.

\section{Inter-organ communication in development:}

\section{Nutrient/oxygen availability is translated into systemic tissue growth via fat body-IPC communication (Figure 1)}

The ability to coordinate cell division, growth and nutrient storage with nutrient availability is mediated via crosstalk between several organs. At the center of this network lies a nutrient sensor/storage organ called the fat body. The fat body is functionally analogous to the vertebrate liver and adipose tissue and it stores and releases energy in response to the energy demands of the insect (Britton and Edgar 1998). In addition to their storage function, fat body cells also act as a nutrient sensor, and not only control the synthesis and utilization of triglycerides and glycogen, but also communicate nutrient availability to the insulin-producing cells (IPCs) in the brain (Colombani et al. 2003). The IPCs then secrete insulin-like peptides (Ilps) 2, 3 
and 5 to increase systemic growth via activation of insulin signalling in peripheral tissues (Ikeya et al. 2002; Geminard, Rulifson, and Leopold 2009). Fat body cells also release IGF-like factor Ilp6 in a Foxo-dependent manner upon cessation of growth triggered by ecdysone (Slaidina et al. 2009). Interestingly, the Ilp6 mutant exhibits a reduction in adult body size, which occurs during post-feeding stages of development, suggesting that the main role of Ilp6 is in regulating post-feeding growth (Slaidina et al. 2009; Okamoto et al. 2009). Recently, Ilp6 has been shown to be involved in the immune response. Upon infection, upregulation of Toll signalling reduces circulating Ilp6 levels to halt growth and allow nutrients to be redirected towards fighting infection (Suzawa et al. 2019). However, how Toll regulates Ilp6 at the translational level remains to be explored.

Fat body mainly regulates IPCs secretion of Ilps via adipokines. Unpaired-2 (Upd-2), a leptin-like cytokine, and the hormone CCHamid2 (which activates a G proteincoupled receptor CCHa2-R) are secreted by fat cells in response to availability of sugar and fat (Rajan and Perrimon 2012; Sano et al. 2015). Other fat body-derived mitogens include GBP1 and 2 (Koyama and Mirth 2016), EGF-like cytokines (without known receptors) induced by dietary sugar, fat and amino acids. TGFbeta/Activin-like ligand Dawdle is also secreted by the fat body upon availability of dietary sugars. It acts through Baboon/Punt receptors and Smad signalling in the fat body to orchestrate overall changes in metabolism, such as triacylglycerol, glucose, glycogen storage and hemolymph $\mathrm{pH}$, as well as release of Ilps in response to food (Ghosh and O'Connor 2014; Bai et al. 2013). Furthermore, insulinotropic peptide Stunted (Sun), secreted from the fat body, acts on heterotrimeric G-protein-coupled receptor (GPCR) Methuselah (Mth) in the IPCs to activate Ilp secretion and larval growth (Delanoue et al. 2016). Several recent studies have uncovered mechanisms by which fat body-derived factors negatively regulate IPCs' Ilps secretion, which in turn affect body size. Eiger (Egr), a tumour necrosis factor (TNF) family cytokine, is released from the fat body surface through induction of TNF $\alpha$-converting enzyme 
(TACE), which cleaves Egr from the membrane when amino acid levels are low (Agrawal et al. 2016). Egr then activates c-Jun N-terminal kinase (JNK) signalling in the IPCs via its receptor Grindelwald (Grnd) to regulate Ilps secretion. Additional mechanisms are also in place to fine tune systemic growth to buffer against changes in circulating insulin. These include secreted decoy of InR (SDR), which is synthesized by glia cells and is secreted into the hemolymph to inhibit Ilps function (Okamoto et al. 2013), and Imaginal morphogenesis protein-Late 2 (ImpL2), that binds to Ilps and is secreted by the fat body under starved conditions to dampen insulin signalling and enhance animal survival (Honegger et al. 2008). Together, these secreted regulators from the fat body (and glia, see next section), positively or negatively regulate Ilps secretion; however, the fashion by which they are coordinated or whether they act redundantly remains unclear.

In addition to sensing nutrient availability, the fat body has recently been shown to be an effective sensor of oxygen levels through HIF-1, a prolyl hydroxylase. Upon sensing a shortage of oxygen, the fat body rapidly shuts down TORC1 signalling, accumulates lipid droplets and relays its hypoxic status through release of HIF-1adependent factors to inhibit Ilps secretion from the brain, thereby restricting systemic growth (Lee, Barretto, and Grewal 2019; Texada et al. 2019). Interestingly, this putative secreted factor lies upstream of the known mediators of fat body/IPC crosstalk, but its identity is so far unknown.

\section{Fat body/Glia/Neural stem cell crosstalk controls overall brain size}

In addition to the fat body/IPC communication axis, the fat body acts as an amino acid sensor and relays nutrient status of the animal to regulate stem cell behaviour in the developing central nervous system (CNS). Upon detection of amino acid availability (Britton and Edgar 1998), a fat body-derived mitogen is released downstream of TORC1 activation (Sousa-Nunes, Yee, and Gould 2011). This signal triggers the release of Ilps 2, 3 and 5 from glial cells that form the microenvironment surrounding neural stem cells (NSCs), to drive NSC cell cycle entry from quiescence (Sousa-Nunes, Yee, and Gould 2011; Chell and Brand 2010) and commencement of 
post-embryonic neurogenesis. NSC reactivation in turn induces glia membrane expansion and remodelling to allow the elaboration of a structural niche around each neural stem cell lineage, which is required to prevent neuronal cell death (Speder and Brand 2018). This sophisticated relay between fat body, glia niche and NSCs ensures that NSCs only re-enter into the cell cycle to initiate larval neurogenesis once the fat body detects amino acid availability, thus coupling stem cell reactivation with overall growth of the organism. Recently, single cell transcriptomic studies showed that while starvation had little effects on cellular composition of the brain, transcription of genes involved in fatty acid metabolism was altered in glia cells (Brunet Avalos et al. 2019), suggesting that metabolic rewiring in the niche occurs in response to nutrient stress, and may possibly in turn regulate neural stem cell proliferation. It is unclear, however, whether the dietary amino acid-induced fat body signal, which activates glia Ilps secretion, is similar or different to the already identified factors implicated in fat body/IPC crosstalk. While Ilps promote systemic growth and storage of fats and sugars, their role regulating the glia niche should be further explored.

Once critical weight (size upon which metamorphosis can be initiated in the absence of further nutritional input) is reached (Mirth, Truman, and Riddiford 2009), NSC proliferation becomes uncoupled from dietary nutrients and glia niche-derived Ilps (Cheng et al. 2011). Instead, activation of Phosphatidylinositol 3-kinase (PI3K) under both fed and starved conditions is achieved through an alternative receptor tyrosine kinase, the Anaplastic Lymphoma Kinase, via the glia-secreted, nutrient-independent ligand Jellybelly (Jeb) (Cheng et al. 2011), therefore uncoupling neurogenesis from nutrient availability. This strategy ensures that the correct number and types of neurons are made by NSCs once the organism develops past critical weight, irrespective of nutrient availability. Other niche derived signals that instruct NSC cell cycle entry from quiescence include Trol (Datta 1995; Caldwell and Datta 1998; Voigt et al. 2002), Anachronism (Ana) (Ebens et al. 1993), Hedgehog (Hh) and Branchless (Bnl) (Park et al. 2003). However, how these signals intersect with Ilps to determine whether NSCs stay quiescent or commence proliferation remains unclear. 


\section{Maturation signals and muscle-derived factors coordinate organ scaling}

In addition to environmental signals, developmental maturation signals are also required to coordinate cell divisions with organism growth. The hormone ecdysone is secreted from the prothoracic gland to orchestrate developmental transitions, such as molting and metamorphosis, with cessation of growth at the end of larval life (reviewed by (Thummel 1996)). Ecdysone also lies upstream of signals that regulate growth such as Ilp6 and the transcription factor dMyc (Delanoue, Slaidina, and Leopold 2010; Slaidina et al. 2009)

Upon injury or tumour growth in the imaginal discs, attainment of metamorphosis is delayed (Colombani, Andersen, and Leopold 2012; Garelli et al. 2012). This ensures additional developmental time for repair to occur. In addition, the growth of other tissues is also slowed down to ensure that relative proportions between the injured/overgrowing disc and the unperturbed discs are maintained. This coordination is achieved via secretion of Ilp8, a relaxin/insulin/IGF-like molecule, from the injured disc (Colombani, Andersen, and Leopold 2012; Garelli et al. 2012). Ilp8 binds to leucine rich repeat-containing G-protein-coupled receptor 3 (Lgr3) in only four neurons in the brain, which in turn regulates the prothoracicotropic hormone (PTTH)producing neurons and the IPCs (Garelli et al. 2012; Vallejo et al. 2015; Colombani et al. 2015). The action of Ilp8 on PTTH-producing neurons results in a delay in ecdysone biosynthesis and metamorphosis. Independently of ecdysone, Ilp8 also slows down the growth of other organs via reduced Ilp3 and Ilp5 regulation in IPCs, which directly acts on insulin/FOXO signalling (Vallejo et al. 2015).

Scaling of organs and appendages with overall size of the animal has recently been shown to be achieved via several mechanisms centered on the BMP/TGF $\beta$ signalling pathway. Activin $\beta$ mutants, which are small in size, have disproportionately small somatic muscles in comparison with imaginal discs, salivary gland and fat body. 
Motoneuron-derived Activin $\beta$ positively regulates muscle mass and body scaling via the transcription factor dSmad2 (Moss-Taylor et al. 2019). Finally, impaired mitochondrial function in the muscle has recently been shown to also cause mitochondrial dysfunction in the fat body, resulting in triglyceride accumulation. This synchronous mitochondrial dysfunction is coordinated via secreted Activin $\beta$ ligand from damaged muscles (Song et al. 2017) to regulate lipid storage in the fat body.

\section{Inter-organ communication in tumourigenesis:}

\section{The cellular immune system positively and negatively regulates tumour growth}

\section{(Figure 2)}

So far, we have discussed how organs communicate to coordinate their size, and couple growth of individual organs with overall nutritional, oxygen and fitness status of the animal. In a similar fashion, tumour cells also interact locally with the immune system and remotely with other organs. These interactions may result in the elimination of the tumour or may facilitate tumour development by allowing diversion of existing physiology to encourage tumour growth. Drosophila has been extensively utilised to study tumourigenesis (Richardson and Portela 2018; Enomoto, Siow, and Igaki 2018) and its genetic tractability makes it an excellent model to investigate the non-autonomous regulation of tumour growth. Most of the evidence on the interactions between tumours and the immune system in Drosophila has been accumulated studying tumours caused by the loss of the so-called 'polarity tumour suppressor genes'. These genes, lethal giant larvae (lgl), scribbled (scrib) and discs large ( $\mathrm{dlg}$ ), have been shown to play important roles in maintaining epithelial cell polarity both in flies and humans. Disruption of cell polarity is one of the hallmarks of human epithelial cancers (Halaoui and McCaffrey 2015). Consistently, Drosophila epithelial tissues mutant for any of these polarity genes display neoplastic growth characterized by loss of tissue architecture, hyperproliferation and invasive behaviour. However, when these polarity-deficient cells exist as a clone in an otherwise normal 
tissue, they are eliminated by the surrounding wildtype cells in a conserved process known as cell competition (reviewed in (Fahey-Lozano et al. 2019; Richardson and Portela 2018; Maruyama and Fujita 2017)). These mutant cells undergo apoptosis via JNK signalling pathway and the evolutionary conserved stress-induced MAPK cascade, as these cells are deemed less fit than their neighbouring wildtype cells. However, when combined with an activated form of Ras (Ras ${ }^{\mathrm{V} 12}$, a gain of function mutation frequently found in human cancers (Hobbs, Der, and Rossman 2016)), these polarity-deficient cells become comparatively fitter than their neighbours, and as a result, apoptosis is inhibited and neoplastic growth is reinstated. Activation of JNK signalling in the presence of Ras ${ }^{\mathrm{V} 12}$ in polarity-deficient cells has been shown to be responsible for many of the neoplastic features of these tumours such as promotion of cell migration and invasion through production of basement membrane degrading matrix metalloproteases (MMPs) (Fahey-Lozano et al. 2019; Richardson and Portela 2018). A large body of evidence exists to demonstrate how non-cell autonomous interactions between tumour cells and their immediate microenvironment within the epithelial tissue play a key role in determining how tumours behave. More recently, however, it has become increasingly clear that longer-range interaction and interorgan crosstalk also play a major role in tumour initiation and progression.

A picture is emerging from research in the past years suggesting that Drosophila innate immune system, much like the mammalian one, evolved not only to protect the animal from pathogens, but also to respond to cell transformation. In different tumour types and in different tissues, extensive interactions between tumour cells and the innate immune system have been shown (Pastor-Pareja, Wu, and Xu 2008; Cordero et al. 2010; Hauling et al. 2014; Parisi et al. 2014; Perez, Lindblad, and Bergmann 2017; Villegas et al. 2018; Araki et al. 2019; Parvy et al. 2019).

It is well established that the mammalian immune system can play a tumourpromoting or tumour-suppressing role, depending on the context (Gonzalez, Hagerling, and Werb 2018; Janssen et al. 2017; Hagerling, Casbon, and Werb 2015), 
and the same holds true in Drosophila. The fly innate immune system, which shares many similarities with its mammalian counterpart, consists of a cellular and a humoral component. Cellular reactions are mediated by hemocytes, the majority of which are macrophage-like cells called plasmatocytes and platelet-like crystal cells that carry out phagocytosis and encapsulation (Govind 2008). The association of hemocytes with polarity-deficient tumours is mostly mediated by damage to the basement membrane. Overexpression of MMPs appears to be sufficient to attract hemocytes, even in the absence of tumours (Pastor-Pareja, Wu, and Xu 2008; Cordero et al. 2010). In scrib or $d l g$ mutant larvae, ablation of the hemocyte population results in larger tumour size while increase in the number of hemocytes results in smaller tumours, suggesting that the immune system can indeed function in restricting tumour growth (Pastor-Pareja, Wu, and Xu 2008; Parisi et al. 2014). In single scrib, lgl or dlg mutant tumours, hemocyte-secreted Egr (Drosophila orthologue of mammalian TNF) activates JNK signalling, which is necessary for the elimination of polarity-deficient cells via apoptosis (Cordero et al. 2010; Parisi et al. 2014). dlg-deficient tumours have also been shown to release PDGF/VEGF-receptor related (Pvr) ligand, Pvf1, in response to Egr signalling. Pvf1 promotes hemocyte proliferation, thus increasing the number of tumour-suppressing immune cells (Parisi et al. 2014). These amplification loops result in a significant increase in circulating hemocytes and trigger a systemic humoral response to restrict tumour growth (see next section). However, similarly to what it is observed for its mammalian orthologue, TNF Egr's effect on tumourigenesis is very much context dependent (Cruceriu et al. 2020; Montfort et al. 2019; Tse, Scott, and Russell 2012). In the presence of Ras ${ }^{\mathrm{V} 12}$, the anti-tumour role of hemocyte-secreted Egr is converted to a pro-tumour one. In this context, Egr is required for JNK mediated tumour cell proliferation and invasion. Strikingly, neoplastic scrib-, Ras ${ }^{V 12}$ tumours generated in an egr loss of function background fail to overgrow, but can simply be restored by transfusion of egr ${ }^{+}$hemocytes (Cordero et al. 2010). Moreover, scrib-, Ras $^{V 12}$ tumours display JNK-dependent increase in reactive oxygen species (ROS) and attract more Egr-secreting hemocytes, which in turn increase JNK induced overproliferation (Perez, Lindblad, and Bergmann 2017). 
In addition, the inflammation mediators NOS (nitric oxide synthase) and lipoxygenase (LOX) are both activated in tumours caused by the oncogenic cooperation of Notch and PI3K signalling and have been shown to mediate hemocyte recruitment (Villegas et al. 2018).

\section{Tumour-suppressive humoral immune response to tumours by the fat body}

The humoral immune response in Drosophila is mainly mediated by two conserved signalling patways: the Toll and Imd pathways in the fat body, the major immune and lipid storage organ of the fly (Govind 2008). $d g^{-}$tumour-induced hemocytes release Spatzle (Spz) and activate Toll signalling in the fat body (Parisi et al. 2014). Similarly, scrib- tumour cells also promote secretion of the cytokines Unpaired 1-3 (Upd1-3), which activate JAK-STAT signalling, to promote addition rounds of hemocyte proliferation and cytokine release (Pastor-Pareja, Wu, and Xu 2008). During an infection, in response to the activation of Toll and Imd pathways, antimicrobial peptides (AMPs) are produced by the fat body (Govind 2008). Among these, Defensin has been shown to also have a direct role in the systemic response to tumours. Defensin recognises phosphatidylserine (PS) exposed on the membrane of polarity-deficient cells and helps to kill them (Parvy et al. 2019). Similarly, in the salivary gland, where expression of Ras ${ }^{\mathrm{V} 12}$ causes basement membrane degradation, this damage recruits hemocytes, exposes PS, triggers activation of Toll and Imd pathways, which in turn induces fat body AMP secretion (Hauling et al. 2014). Fat body-derived AMPs have also been recently shown to restrict hematopoietic tumour growth caused by a mutation in the multi sex combs ( $m x c)$ gene (Araki et al. 2019). Together, these studies suggest that AMP-mediated humoral response might represent an important mechanism of tumour suppression in Drosophila. Interestingly, AMPs are regarded as potential cancer therapeutic agents as they have been shown to have antitumoral activity both in vitro and in vivo, probably by targeting PS, which is abundant on the external membrane of cancer cells (Deslouches and Di 2017; Yavari et al. 2018). 


\section{Tumours interact with the tracheal system}

To be able to sustain growing demands of oxygen and nutrients, tumours interact with the vasculature and stimulate it to produce new vessels, a process called angiogenesis (Muz et al. 2015). In Drosophila, oxygen and other gasses are transported throughout the body by a network of branched tubular structures called the tracheal system (Hayashi and Kondo 2018). Much like human malignances, Drosophila lgl-, Ras ${ }^{V 12}$ tumours suffer from oxygen shortage. In response to this hypoxic stress, the growth of ectopic branches as well as other mechanisms typical of mammalian angiogenesis, such as vascular cooption (Zhang, Wang, and Dudley 2020) and vasculogenic mimicry (Luo et al. 2020), have been observed in these tumours (Grifoni et al. 2015). In vascular cooption, tumour cells migrate along the vasculature to eventually embed the vessels within the tumour mass. In around half of the tumours, cells were also found to express the tracheal fate determinant Trachealess (Trh) and to form de novo tracheal tubes as well as mosaic tubes within the existing network, a process akin to mammalian vasculogenic mimicry (Grifoni et al. 2015). Similarly, Ras/Src tumours, which become neoplastic when exposed to a high sugar diet, give rise to metastasis that tightly associate with the tracheal system and express Trh (Hirabayashi, Baranski, and Cagan 2013). Finally, the close interaction between disseminating tumour cells and the tracheal system has also been observed in gut tumour models (Bangi et al. 2016; Campbell et al. 2019), suggesting that this might be a common feature of epithelial transformation in Drosophila.

\section{Tumour-induced reprogramming of host metabolism}

Metabolic reprogramming is nowadays a well-established hallmark of cancer. However, the origins of the understanding that metabolism is altered in cancer cells can be traced back to nearly a century ago, when Otto Warburg described that tumour cells switch from oxidative phosphorylation to anaerobic glycolysis, even in the presence of oxygen, and convert glucose into lactate through a process called “Warburg effect” (Warburg 1925). Since then, many metabolic dysfunctions associated with tumours have been described, and interest in cancer metabolism has 
grown considerably in recent years. Studies have mostly concentrated on gaining an understanding of how cancer metabolism is regulated, how it influences disease progression and how it can be exploited for cancer therapy. However, given the heterogeneity among tumour types, and even within a single tumour, a single cancer metabolic signature does not exist (Cantor and Sabatini 2012). The high energetic demands of tumours have been shown to not only influence the metabolic state of their local microenvironment, but to also alter the metabolism of the host. In this section of the review, we will summarise findings from Drosophila studies that shed light on the host-tumour metabolic interplay.

\section{Nutritional cues modulate tumour growth}

Tumours display high demand for certain nutrients to sustain their rapid growth and proliferation. It has been shown that Ras/Src-transformed cells which are normally eliminated from the epithelium, generate highly proliferative, metastatic tumours when flies are raised on high-sugar diets. In contrast to wildtype tissues that display insulin resistance, Ras/Src-transformed cells increase Wnt/wingless signalling in response to high sugar to enhance insulin signalling and insulin sensitivity, which in turn results in their increased proliferation (Hirabayashi, Baranski, and Cagan 2013). Interestingly, nutrient-independent tumour growth is observed both in Drosophila mutants for PTEN (a negative regulator of phosphatidylinositol-3-kinase (PI3K)), and in mouse xenografts of tumours that constitutively activate PI3K (Nowak et al. 2013; Kalaany and Sabatini 2009). Under nutrient restriction, PTEN-deficient cells switch from hypertrophic growth to hyperplastic growth. This proliferative advantage is dependent on the activity of the downstream component TOR and the amino acid transporter Slimfast (Slif). In addition, the size of neighbouring and distal tissues is reduced, suggesting PTEN-mutant cells outcompete other tissues for resources to support their own growth (Nowak et al. 2013). Thus, activation of the PI3K pathway is a strategy which enables tumour growth to be uncoupled from nutrition, therefore conferring a growth advantage to these tumours at the expense of other tissues. 
Energetic demands of tumours are met by utilization of amino acids and their metabolic intermediates such as ATPs and acetyl-CoA (Vettore, Westbrook, and Tennant 2020). However, these metabolic demands are not uniform and appear to be highly dependent on the nature of the tumour's mutations. Recently, the effects of particular amino acids on tumour growth have been investigated. Decreasing dietary histidine has been shown to strongly inhibit the growth of Myc-dependent dedifferentiation tumours in the Drosophila CNS. Tumours induced by loss of the transcription factor Nerfin-1 or gain of Notch, that are highly dependent on Myc upregulation and increased cellular growth, are found to be sensitive to histidine depletion; whereas dietary histidine withdrawal has little effect on wildtype NSCs and prospero mutant neural tumours, which are less dependent on them (Froldi et al. 2019), suggesting Myc-dependency may render tumours specifically dependent on histidine. In the future, a better understanding of how tumour's genetic signature is linked to its metabolic dependency will be fundamental for defining and targeting metabolic vulnerabilities in cancer.

\section{Autophagy as a regulator of tumour growth}

Autophagy, an intracellular catabolic process that eliminates aberrant and unnecessary cytoplasmic material, has been highlighted as a non-cell autonomous regulator of tumour growth. Inhibition of autophagy in Ras-activated wing epithelial tissues results in cell overgrowth, mediated by the induction of oxidative stress and activation of JNK pathway. Its inhibition also causes non-cell autonomous effects by activating autophagy, cell death and cell proliferation in the surrounding wildtype tissue (Manent et al. 2017). Conversely, another study showed that scrib-, Ras ${ }^{V 12}$ tumourinduced ROS triggered autophagy both locally and in distant tissues to sustain tumour proliferation. Strikingly, inhibition of autophagy in the surrounding tissue strongly reduced tumour growth and invasion (Katheder et al. 2017). In addition, active amino acid import mediated by the amino acid transporter Slif also played a role in sustaining tumour growth (Katheder et al. 2017). Together, these reports showed that autophagy generated nutrients via degradation of surrounding tissues to 'feed' 
tumours. Given the apparent conservation of autophagy mechanisms between flies and mammals, further work in Drosophila might help elucidate how metabolic alterations in the tumour microenvironment and other distant tissues can in turn influence tumour growth.

\section{Drosophila models of cancer-associated cachexia (Figure 3)}

The effects of tumours on distal tissues have recently been investigated in the context cancer-associated cachexia (CAC). Cachexia is a chronic wasting condition that causes dramatic involuntary weight loss and systemic dysfunction (Tisdale 2008; Fearon et al. 2011; Argiles et al. 2014). The most recognised hallmark of CAC is loss of skeletal muscle, which is caused by increased protein degradation and decreased protein synthesis. However, loss of adipose tissue has also be observed in many cases (Tisdale 2008; Fearon et al. 2011). Most of the studies on cachexia have been performed in rodents, however, recently, three independent studies have proved the utility of Drosophila in this field of research (Figueroa-Clarevega and Bilder 2015; Kwon et al. 2015; Song et al. 2019).

All three studies have been performed in adult flies. The first study transplanted larval $s c r i b^{-}, R^{V} s^{V 12}$ tumours into wildtype adult flies, that induced wasting of distal organs and an overall reduction of host fitness. Tumour-bearing animals displayed degeneration of thoracic muscles and ovaries and considerable reduction of fat body mass (Figueroa-Clarevega and Bilder 2015). The second study utilised a constitutively activated version of Yorkie $\left(y k i^{a c t}\right)$ in intestinal stem cells (ISC), which induced tumours in the midgut, and systemic wasting of ovary, fat body and muscle (Kwon et al. 2015). Both $s c r i b-, R^{V} s^{V 12}$ and $y k^{a c t}$ tumours were found to secrete high levels of ImpL2, a homologue of the human insulin growth factor-binding protein (IGFBP). IGFBP is known to antagonises IGFs and reduce systemic insulin signalling, as indicated by increased circulating sugar levels (hyperglycaemia), suggesting that tumour-induced ImpL2 upregulation resulted in insulin resistance of peripheral tissues. Furthermore, ImpL2 inactivation in the midgut was able to rescue ovary wasting and muscle 
degeneration, and ImpL2 expression in the absence of tumour was also sufficient to induce CAC (Figueroa-Clarevega and Bilder 2015; Kwon et al. 2015). A third study found that Yki-induced tumours in addition to IMPL2, autonomously promoted tumour growth via Egfr/MEK signalling. Tumours secrete PVR ligand Pvf1 to nonautonomously activate aberrant Pvr/MEK signalling, which in turn induced wasting in distal tissues. The effect of Pvf1 is independent of that of ImpL2, suggesting multiple secreted factors may be involved in mediating CAC (Song et al. 2019).

Aiming to investigate the poorly understood mechanisms of tumour-host interactions, these works have revealed two tumour-derived factors with a systemic effect on host metabolism. These results support the hypothesis that cachexia is not only detrimental for the host, but could also support tumour growth through nutrient mobilisation from distal tissues. Nonetheless, the fact that cachexia also occurs in many other pathologies without a tumour acting as a 'sink' of nutrients (Tisdale 2008) puts forward the question of whether cachexia benefits tumours through other mechanisms. The cachectic Drosophila models will prove useful to answer some of these open questions.

\section{Final remarks}

Important parallels lie between flies and humans in how physiology is regulated, whereby equivalent organs and evolutionarily conserved pathways and enzymes regulate carbohydrate and lipid metabolism. In this review, we outlined recent studies in Drosophila that allowed us to better understand how tissues interact to coordinate scaling and how organ size control is coordinated with the available nutrients and oxygen. Inter-organ cross talks are not merely interesting in the context of organismal growth and organ scaling during development, but are becoming increasingly important in deciphering long-standing, clinical problems such as cancer-associated cachexia. As discussed in this review, increasing evidence suggests tumours might be able to satisfy their autonomous energetic needs by non-autonomously altering the physiology and metabolism of other tissues. Indeed, Drosophila cachexia models and tumour-induced microenvironmental autophagy support the hypothesis that tumour communicates with 
surrounding and peripheral tissues to redirect nutrient resources to support their own growth at the expense of other organs. Additionally, we have presented some important parallels between fly tumours to their human counterparts, and discussed how, for example, tumour growth depends on the intimate interaction between transformed cells and the immune system, which can positively or negatively regulate tumour growth depending on the context.

A powerful set of tools now exists for manipulating fly physiology: in addition to the widely used Gal4 system, alternative expression systems such as the Q system and the LexA sytstem now allow independent spatially- and temporally-controlled expression or knockdown of genes, simultaneously in various tissues or tumours (del Valle Rodriguez, Didiano, and Desplan 2011). Moreover, the growing list of fluorescentlytagged proteins and GFP gene insertions, combined with the ability to perform whole animal imaging (Manning and Doe 2017), enables real-time observation of inter-organ crosstalk.

In order to decipher tumour-host interactions, it is fundamental to unveil the signalling pathways that link tumour growth to host metabolism and immunity. However, the mechanisms arbitrating this crosstalk are still being determined. Drosophila models have proved fruitful for studying these complex intercommunications and these techniques will no doubt continue to help us better understand the dynamic nature of long-range organ interactions both in development and in cancer in the near future.

\section{Acknowledgements:}

We would like to thank Michael Zavortink for critical reading of the manuscript. Figures were created with Biorender.com.

\section{References:}

Agrawal, N., R. Delanoue, A. Mauri, D. Basco, M. Pasco, B. Thorens, and P. Leopold. 2016. 'The Drosophila TNF Eiger Is an Adipokine that Acts on InsulinProducing Cells to Mediate Nutrient Response', Cell Metab, 23: 675-84.

Araki, M., M. Kurihara, S. Kinoshita, R. Awane, T. Sato, Y. Ohkawa, and Y. H. Inoue. 2019. 'Anti-tumour effects of antimicrobial peptides, components of the 
innate immune system, against haematopoietic tumours in Drosophila mxc mutants', Dis Model Mech, 12.

Argiles, J. M., S. Busquets, B. Stemmler, and F. J. Lopez-Soriano. 2014. 'Cancer cachexia: understanding the molecular basis', Nat Rev Cancer, 14: 754-62.

Bai, H., P. Kang, A. M. Hernandez, and M. Tatar. 2013. 'Activin signaling targeted by insulin/dFOXO regulates aging and muscle proteostasis in Drosophila', PLoS Genet, 9: e1003941.

Bangi, E., C. Murgia, A. G. Teague, O. J. Sansom, and R. L. Cagan. 2016. 'Functional exploration of colorectal cancer genomes using Drosophila', Nat Commun, 7: 13615.

Britton, J. S., and B. A. Edgar. 1998. 'Environmental control of the cell cycle in Drosophila: nutrition activates mitotic and endoreplicative cells by distinct mechanisms', Development, 125: 2149-58.

Brunet Avalos, C., G. L. Maier, R. Bruggmann, and S. G. Sprecher. 2019. 'Single cell transcriptome atlas of the Drosophila larval brain', Elife, 8.

Caldwell, M. C., and S. Datta. 1998. 'Expression of cyclin E or DP/E2F rescues the G1 arrest of trol mutant neuroblasts in the Drosophila larval central nervous system', Mech Dev, 79: 121-30.

Campbell, K., F. Rossi, J. Adams, I. Pitsidianaki, F. M. Barriga, L. Garcia-Gerique, E. Batlle, J. Casanova, and A. Casali. 2019. 'Collective cell migration and metastases induced by an epithelial-to-mesenchymal transition in Drosophila intestinal tumors', Nat Commun, 10: 2311.

Cantor, J. R., and D. M. Sabatini. 2012. 'Cancer cell metabolism: one hallmark, many faces', Cancer Discov, 2: 881-98.

Chell, J. M., and A. H. Brand. 2010. 'Nutrition-responsive glia control exit of neural stem cells from quiescence', Cell, 143: 1161-73.

Cheng, L. Y., A. P. Bailey, S. J. Leevers, T. J. Ragan, P. C. Driscoll, and A. P. Gould. 2011. 'Anaplastic lymphoma kinase spares organ growth during nutrient restriction in Drosophila', Cell, 146: 435-47.

Colombani, J., D. S. Andersen, L. Boulan, E. Boone, N. Romero, V. Virolle, M. Texada, and P. Leopold. 2015. 'Drosophila Lgr3 Couples Organ Growth with Maturation and Ensures Developmental Stability', Curr Biol, 25: 2723-9.

Colombani, J., D. S. Andersen, and P. Leopold. 2012. 'Secreted peptide Dilp8 coordinates Drosophila tissue growth with developmental timing', Science, 336: 582-5.

Colombani, J., S. Raisin, S. Pantalacci, T. Radimerski, J. Montagne, and P. Leopold. 2003. 'A nutrient sensor mechanism controls Drosophila growth', Cell, 114: 739-49.

Cordero, J. B., J. P. Macagno, R. K. Stefanatos, K. E. Strathdee, R. L. Cagan, and M. Vidal. 2010. 'Oncogenic Ras diverts a host TNF tumor suppressor activity into tumor promoter', Dev Cell, 18: 999-1011. 
Cruceriu, D., O. Baldasici, O. Balacescu, and I. Berindan-Neagoe. 2020. 'The dual role of tumor necrosis factor-alpha (TNF-alpha) in breast cancer: molecular insights and therapeutic approaches', Cell Oncol (Dordr), 43: 118.

Datta, S. 1995. 'Control of proliferation activation in quiescent neuroblasts of the Drosophila central nervous system', Development, 121: 1173-82.

del Valle Rodriguez, A., D. Didiano, and C. Desplan. 2011. 'Power tools for gene expression and clonal analysis in Drosophila', Nat Methods, 9: 47-55.

Delanoue, R., E. Meschi, N. Agrawal, A. Mauri, Y. Tsatskis, H. McNeill, and P. Leopold. 2016. 'Drosophila insulin release is triggered by adipose Stunted ligand to brain Methuselah receptor', Science, 353: 1553-56.

Delanoue, R., M. Slaidina, and P. Leopold. 2010. 'The steroid hormone ecdysone controls systemic growth by repressing dMyc function in Drosophila fat cells', Dev Cell, 18: 1012-21.

Deslouches, B., and Y. P. Di. 2017. 'Antimicrobial peptides with selective antitumor mechanisms: prospect for anticancer applications', Oncotarget, 8: 46635-51.

Ebens, A. J., H. Garren, B. N. Cheyette, and S. L. Zipursky. 1993. 'The Drosophila anachronism locus: a glycoprotein secreted by glia inhibits neuroblast proliferation', Cell, 74: 15-27.

Enomoto, M., C. Siow, and T. Igaki. 2018. 'Drosophila As a Cancer Model', Adv Exp Med Biol, 1076: 173-94.

Fahey-Lozano, N., J. E. La Marca, M. Portela, and H. E. Richardson. 2019. 'Drosophila Models of Cell Polarity and Cell Competition in Tumourigenesis', Adv Exp Med Biol, 1167: 37-64.

Fearon, K., F. Strasser, S. D. Anker, I. Bosaeus, E. Bruera, R. L. Fainsinger, A. Jatoi, C. Loprinzi, N. MacDonald, G. Mantovani, M. Davis, M. Muscaritoli, F. Ottery, L. Radbruch, P. Ravasco, D. Walsh, A. Wilcock, S. Kaasa, and V. E. Baracos. 2011. 'Definition and classification of cancer cachexia: an international consensus', Lancet Oncol, 12: 489-95.

Figueroa-Clarevega, A., and D. Bilder. 2015. 'Malignant Drosophila tumors interrupt insulin signaling to induce cachexia-like wasting', Dev Cell, 33: 47-55.

Froldi, F., P. Pachnis, M. Szuperak, O. Costas, T. Fernando, A. P. Gould, and L. Y. Cheng. 2019. 'Histidine is selectively required for the growth of Mycdependent dedifferentiation tumours in the Drosophila CNS', Embo j, 38.

Garelli, A., A. M. Gontijo, V. Miguela, E. Caparros, and M. Dominguez. 2012. 'Imaginal discs secrete insulin-like peptide 8 to mediate plasticity of growth and maturation', Science, 336: 579-82.

Geminard, C., E. J. Rulifson, and P. Leopold. 2009. 'Remote control of insulin secretion by fat cells in Drosophila', Cell Metab, 10: 199-207. 
Ghosh, A. C., and M. B. O'Connor. 2014. 'Systemic Activin signaling independently regulates sugar homeostasis, cellular metabolism, and $\mathrm{pH}$ balance in Drosophila melanogaster', Proc Natl Acad Sci U S A, 111: 5729-34.

Gonzalez, H., C. Hagerling, and Z. Werb. 2018. 'Roles of the immune system in cancer: from tumor initiation to metastatic progression', Genes Dev, 32: 1267-84.

Govind, S. 2008. 'Innate immunity in Drosophila: Pathogens and pathways', Insect Sci, 15: 29-43.

Grifoni, D., M. Sollazzo, E. Fontana, F. Froldi, and A. Pession. 2015. 'Multiple strategies of oxygen supply in Drosophila malignancies identify tracheogenesis as a novel cancer hallmark', Sci Rep, 5: 9061.

Hagerling, C., A. J. Casbon, and Z. Werb. 2015. 'Balancing the innate immune system in tumor development', Trends Cell Biol, 25: 214-20.

Halaoui, R., and L. McCaffrey. 2015. 'Rewiring cell polarity signaling in cancer', Oncogene, 34: 939-50.

Hauling, T., R. Krautz, R. Markus, A. Volkenhoff, L. Kucerova, and U. Theopold. 2014. 'A Drosophila immune response against Ras-induced overgrowth', Biol Open, 3: 250-60.

Hayashi, S., and T. Kondo. 2018. 'Development and Function of the Drosophila Tracheal System', Genetics, 209: 367-80.

Hirabayashi, S., T. J. Baranski, and R. L. Cagan. 2013. 'Transformed Drosophila cells evade diet-mediated insulin resistance through wingless signaling', Cell, 154: 664-75.

Hobbs, G. A., C. J. Der, and K. L. Rossman. 2016. 'RAS isoforms and mutations in cancer at a glance', J Cell Sci, 129: 1287-92.

Honegger, B., M. Galic, K. Kohler, F. Wittwer, W. Brogiolo, E. Hafen, and H. Stocker. 2008. 'Imp-L2, a putative homolog of vertebrate IGF-binding protein 7, counteracts insulin signaling in Drosophila and is essential for starvation resistance', J Biol, 7: 10.

Ikeya, T., M. Galic, P. Belawat, K. Nairz, and E. Hafen. 2002. 'Nutrient-dependent expression of insulin-like peptides from neuroendocrine cells in the CNS contributes to growth regulation in Drosophila', Curr Biol, 12: 1293-300.

Janssen, L. M. E., E. E. Ramsay, C. D. Logsdon, and W. W. Overwijk. 2017. 'The immune system in cancer metastasis: friend or foe?', J Immunother Cancer, 5: 79.

Kalaany, N. Y., and D. M. Sabatini. 2009. 'Tumours with PI3K activation are resistant to dietary restriction', Nature, 458: 725-31.

Katheder, N. S., R. Khezri, F. O'Farrell, S. W. Schultz, A. Jain, M. M. Rahman, K. O. Schink, T. A. Theodossiou, T. Johansen, G. Juhasz, D. Bilder, A. Brech, H. Stenmark, and T. E. Rusten. 2017. 'Microenvironmental autophagy promotes tumour growth', Nature, 541: 417-20. 
Koyama, T., and C. K. Mirth. 2016. 'Growth-Blocking Peptides As NutritionSensitive Signals for Insulin Secretion and Body Size Regulation', PLoS Biol, 14: e1002392.

Kwon, Y., W. Song, I. A. Droujinine, Y. Hu, J. M. Asara, and N. Perrimon. 2015. 'Systemic organ wasting induced by localized expression of the secreted insulin/IGF antagonist ImpL2', Dev Cell, 33: 36-46.

Lee, B., E. C. Barretto, and S. S. Grewal. 2019. 'TORC1 modulation in adipose tissue is required for organismal adaptation to hypoxia in Drosophila', Nat Commun, 10: 1878.

Luo, Q., J. Wang, W. Zhao, Z. Peng, X. Liu, B. Li, H. Zhang, B. Shan, C. Zhang, and C. Duan. 2020. 'Vasculogenic mimicry in carcinogenesis and clinical applications', J Hematol Oncol, 13: 19.

Manent, J., S. Banerjee, R. de Matos Simoes, T. Zoranovic, C. Mitsiades, J. M. Penninger, K. J. Simpson, P. O. Humbert, and H. E. Richardson. 2017. 'Autophagy suppresses Ras-driven epithelial tumourigenesis by limiting the accumulation of reactive oxygen species', Oncogene, 36: 5576-92.

Manning, L., and C. Q. Doe. 2017. 'Immunofluorescent antibody staining of intact Drosophila larvae', Nat Protoc, 12: 1-14.

Maruyama, T., and Y. Fujita. 2017. 'Cell competition in mammals - novel homeostatic machinery for embryonic development and cancer prevention', Curr Opin Cell Biol, 48: 106-12.

Mirth, C. K., J. W. Truman, and L. M. Riddiford. 2009. 'The ecdysone receptor controls the post-critical weight switch to nutrition-independent differentiation in Drosophila wing imaginal discs', Development, 136: 2345-53.

Montfort, A., C. Colacios, T. Levade, N. Andrieu-Abadie, N. Meyer, and B. Segui. 2019. 'The TNF Paradox in Cancer Progression and Immunotherapy', Front Immunol, 10: 1818.

Moss-Taylor, L., A. Upadhyay, X. Pan, M. J. Kim, and M. B. O'Connor. 2019. 'Body Size and Tissue-Scaling Is Regulated by Motoneuron-Derived Activin beta in Drosophila melanogaster', Genetics, 213: 1447-64.

Muz, B., P. de la Puente, F. Azab, and A. K. Azab. 2015. 'The role of hypoxia in cancer progression, angiogenesis, metastasis, and resistance to therapy', Hypoxia (Auckl), 3: 83-92.

Nowak, K., G. Seisenbacher, E. Hafen, and H. Stocker. 2013. 'Nutrient restriction enhances the proliferative potential of cells lacking the tumor suppressor PTEN in mitotic tissues', Elife, 2: e00380.

Okamoto, N., R. Nakamori, T. Murai, Y. Yamauchi, A. Masuda, and T. Nishimura. 2013. 'A secreted decoy of InR antagonizes insulin/IGF signaling to restrict body growth in Drosophila', Genes Dev, 27: 87-97.

Okamoto, N., N. Yamanaka, Y. Yagi, Y. Nishida, H. Kataoka, M. B. O'Connor, and A. Mizoguchi. 2009. 'A fat body-derived IGF-like peptide regulates postfeeding growth in Drosophila', Dev Cell, 17: 885-91. 
Parisi, F., R. K. Stefanatos, K. Strathdee, Y. Yu, and M. Vidal. 2014. 'Transformed epithelia trigger non-tissue-autonomous tumor suppressor response by adipocytes via activation of Toll and Eiger/TNF signaling', Cell Rep, 6: 855-67.

Park, Y., C. Rangel, M. M. Reynolds, M. C. Caldwell, M. Johns, M. Nayak, C. J. Welsh, S. McDermott, and S. Datta. 2003. 'Drosophila perlecan modulates FGF and hedgehog signals to activate neural stem cell division', Dev Biol, 253: 247-57.

Parvy, J. P., Y. Yu, A. Dostalova, S. Kondo, A. Kurjan, P. Bulet, B. Lemaitre, M. Vidal, and J. B. Cordero. 2019. 'The antimicrobial peptide defensin cooperates with tumour necrosis factor to drive tumour cell death in Drosophila', Elife, 8.

Pastor-Pareja, J. C., M. Wu, and T. Xu. 2008. 'An innate immune response of blood cells to tumors and tissue damage in Drosophila', Dis Model Mech, 1: 14454; discussion 53.

Perez, E., J. L. Lindblad, and A. Bergmann. 2017. 'Tumor-promoting function of apoptotic caspases by an amplification loop involving ROS, macrophages and JNK in Drosophila', Elife, 6.

Rajan, A., and N. Perrimon. 2012. 'Drosophila cytokine unpaired 2 regulates physiological homeostasis by remotely controlling insulin secretion', Cell, 151: 123-37.

Richardson, H. E., and M. Portela. 2018. 'Modelling Cooperative Tumorigenesis in Drosophila', Biomed Res Int, 2018: 4258387.

Sano, H., A. Nakamura, M. J. Texada, J. W. Truman, H. Ishimoto, A. Kamikouchi, Y. Nibu, K. Kume, T. Ida, and M. Kojima. 2015. 'The Nutrient-Responsive Hormone CCHamide-2 Controls Growth by Regulating Insulin-like Peptides in the Brain of Drosophila melanogaster', PLoS Genet, 11: e1005209.

Slaidina, M., R. Delanoue, S. Gronke, L. Partridge, and P. Leopold. 2009. 'A Drosophila insulin-like peptide promotes growth during nonfeeding states', Dev Cell, 17: 874-84.

Song, W., S. Kir, S. Hong, Y. Hu, X. Wang, R. Binari, H. W. Tang, V. Chung, A. S. Banks, B. Spiegelman, and N. Perrimon. 2019. 'Tumor-Derived Ligands Trigger Tumor Growth and Host Wasting via Differential MEK Activation', Dev Cell, 48: 277-86.e6.

Song, W., E. Owusu-Ansah, Y. Hu, D. Cheng, X. Ni, J. Zirin, and N. Perrimon. 2017. 'Activin signaling mediates muscle-to-adipose communication in a mitochondria dysfunction-associated obesity model', Proc Natl Acad Sci U $S$ A, 114: 8596-601.

Sousa-Nunes, R., L. L. Yee, and A. P. Gould. 2011. 'Fat cells reactivate quiescent neuroblasts via TOR and glial insulin relays in Drosophila', Nature, 471: 508-12. 
Speder, P., and A. H. Brand. 2018. 'Systemic and local cues drive neural stem cell niche remodelling during neurogenesis in Drosophila', Elife, 7.

Suzawa, M., N. M. Muhammad, B. S. Joseph, and M. L. Bland. 2019. 'The Toll Signaling Pathway Targets the Insulin-like Peptide Dilp6 to Inhibit Growth in Drosophila', Cell Rep, 28: 1439-46.e5.

Texada, M. J., A. F. Jorgensen, C. F. Christensen, T. Koyama, A. Malita, D. K. Smith, D. F. M. Marple, E. T. Danielsen, S. K. Petersen, J. L. Hansen, K. A. Halberg, and K. F. Rewitz. 2019. 'A fat-tissue sensor couples growth to oxygen availability by remotely controlling insulin secretion', Nat Commun, 10: 1955.

Thummel, C. S. 1996. 'Flies on steroids--Drosophila metamorphosis and the mechanisms of steroid hormone action', Trends Genet, 12: 306-10.

Tisdale, M. J. 2008. 'Catabolic mediators of cancer cachexia', Curr Opin Support Palliat Care, 2: 256-61.

Tse, B. W., K. F. Scott, and P. J. Russell. 2012. 'Paradoxical roles of tumour necrosis factor-alpha in prostate cancer biology', Prostate Cancer, 2012: 128965.

Vallejo, D. M., S. Juarez-Carreno, J. Bolivar, J. Morante, and M. Dominguez. 2015. 'A brain circuit that synchronizes growth and maturation revealed through Dilp8 binding to Lgr3', Science, 350: aac6767.

Vettore, L., R. L. Westbrook, and D. A. Tennant. 2020. 'New aspects of amino acid metabolism in cancer', Br J Cancer, 122: 150-56.

Villegas, S. N., R. Gombos, L. Garcia-Lopez, I. Gutierrez-Perez, J. Garcia-Castillo, D. M. Vallejo, V. G. Da Ros, E. Ballesta-Illan, J. Mihaly, and M. Dominguez. 2018. 'PI3K/Akt Cooperates with Oncogenic Notch by Inducing Nitric Oxide-Dependent Inflammation', Cell Rep, 22: 2541-49.

Voigt, A., R. Pflanz, U. Schafer, and H. Jackle. 2002. 'Perlecan participates in proliferation activation of quiescent Drosophila neuroblasts', Dev Dyn, 224: 403-12.

Warburg, Otto. 1925. 'The Metabolism of Carcinoma Cells', The Journal of Cancer Research, 9: 148-63.

Yavari, B., R. Mahjub, M. Saidijam, M. Raigani, and M. Soleimani. 2018. 'The Potential Use of Peptides in Cancer Treatment', Curr Protein Pept Sci, 19: 759-70.

Zhang, Y., S. Wang, and A. C. Dudley. 2020. 'Models and molecular mechanisms of blood vessel co-option by cancer cells', Angiogenesis, 23: 17-25. 


\section{Figure legends}

\section{Figure 1}

Organ growth is coordinated with systemic body size control via inter-organ crosstalk

The fat body (light brown) acts as a nutrient sensor and relays nutrient availability information to IPCs (orange) via secreted signals such as Upd-2, GBPs, CCHamid2, Dawdle, ImpL2, Egr and Sun, to regulate Ilps 2,3,5 secretion which activates systemic growth. Other mediators of fat body, muscle and wing discs are as depicted in the diagram, and explained in more details in the text.

Fat body and brain crosstalk (inset). The fat body senses nutrient availability and sends a not yet identified signal to the glia cells (gray) in the brain. Glia cells produce Ilps 2,3,5, which trigger NSC (red) exit from quiescence and cell growth and division. Later in development, neurogenesis becomes nutrient-independent and NSC proliferation relies on glia cell secretion of Jeb ligand. See text for more details.

\section{Figure 2}

Polarity deficient tumours and immune system crosstalk. A. Tumour suppressive interactions: Hemocytes (gray) are recruited to tumours caused by loss of cell polarity genes (scrib- or $\mathrm{dlg}^{-}$, green) and secrete Egr which activates JNK signaling in tumour cells. Several of the outputs of JNK activation, including the release of secreted factors such as Pvf1 and Upds, promote further hemocyte recruitment and proliferation as well as a systemic humoral response mediated by the fat body (light brown). See text for more details. These interactions with the immune system ultimately contribute to the elimination of tumour cells via apoptosis. B. Tumour promoting interactions: In the presence of constitutive activation of the oncogene Ras, apoptosis in the polarity deficient cells ( $s c r i b-, \operatorname{Ras}^{V 12}$, green) is inhibited and the tumour suppressive function of the hemocytes (gray) is converted to tumour promoting. In this context, hemocyte-secreted Egr promotes JNK-mediated tumour proliferation and invasion. See text for more details.

\section{Figure 3}

Drosophila models of cancer-associated cachexia: ImpL2 and Pvf1 induce systemic organ wasting in two different tumour types. ImpL2 and Pvf1 secreted by Yki-overxpressing (green, left) or $s c r i b-{ }^{-} \operatorname{Rs}^{V 12}$ (green, right) tumour cells are responsible for inducing wasting of muscle (pink), fat body (light brown) and ovaries (purple), by inducing insulin resistance and aberrant Pvr/MEK activation, respectively. See text for more details. 


\section{Fat body}

Lipid Upd-2 GBPs Cappose

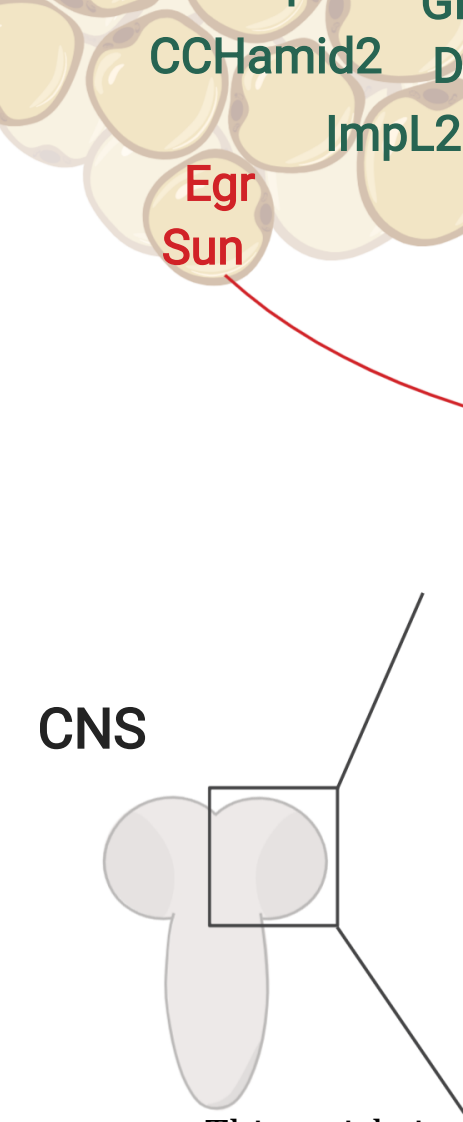

This article is protected by copyright. All rights reserved.

Wing imaginal disc

\section{Glia cells}

Systemic growth

Ilps 2,3,5

Ilps 2,3,5

Activin $\beta$

Myoglianin

Muscle
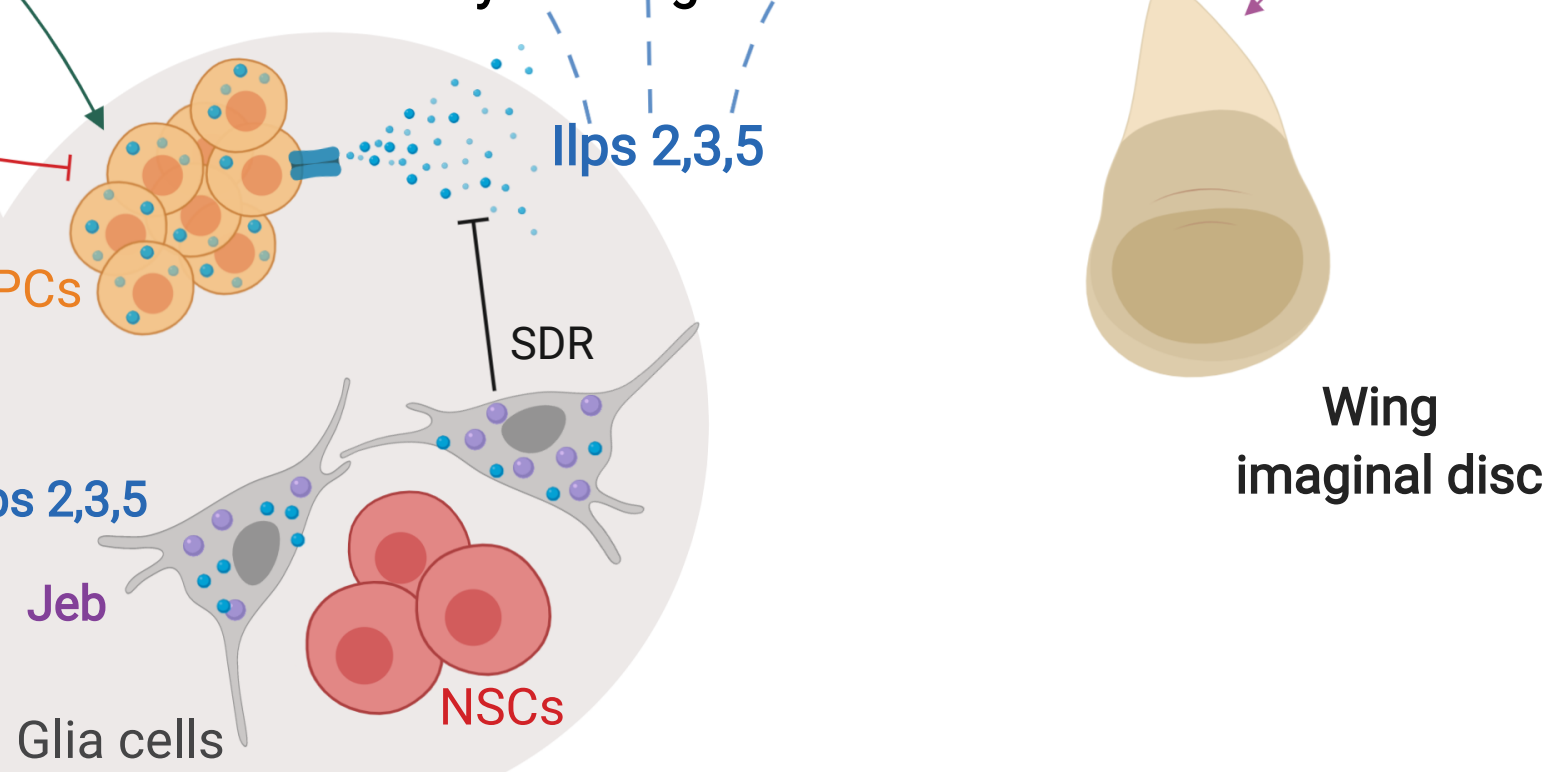


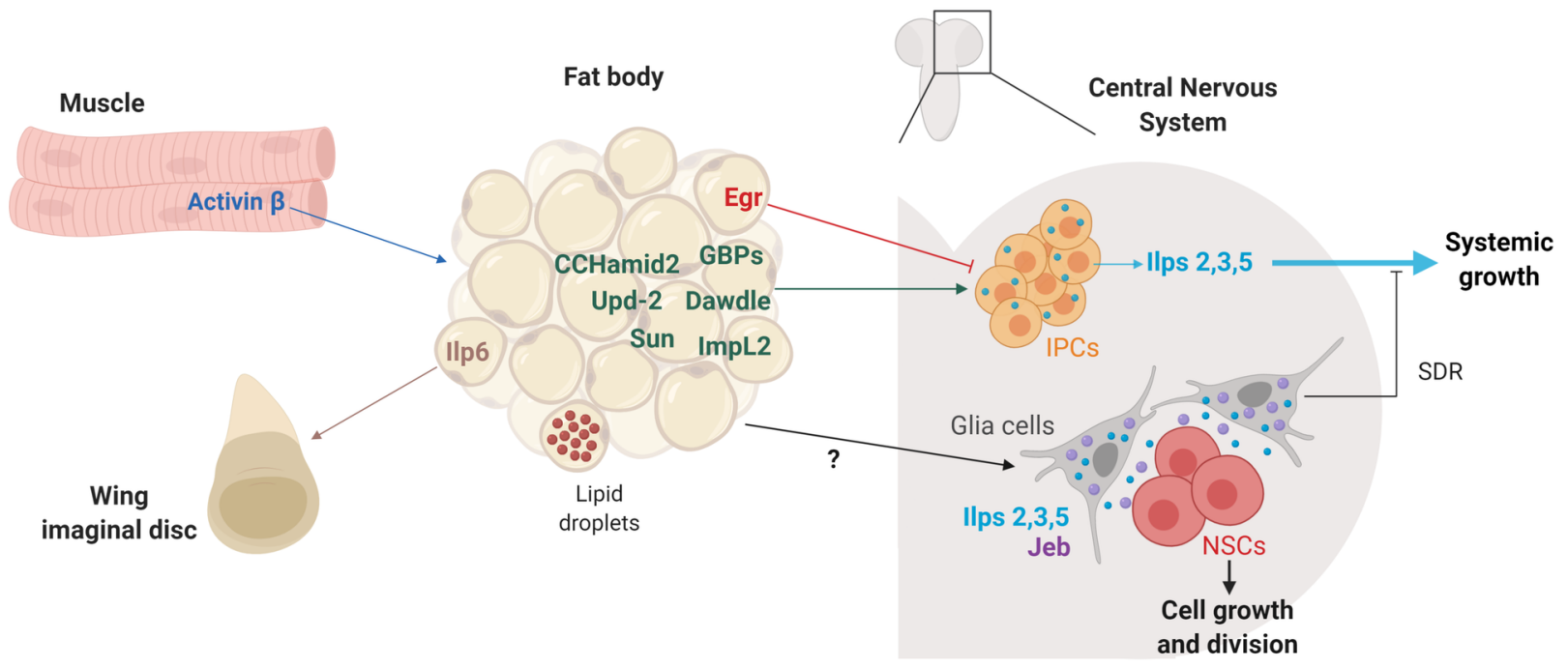

WDEV_394_Figure 1_v2.tif

This article is protected by copyright. All rights reserved. 
A

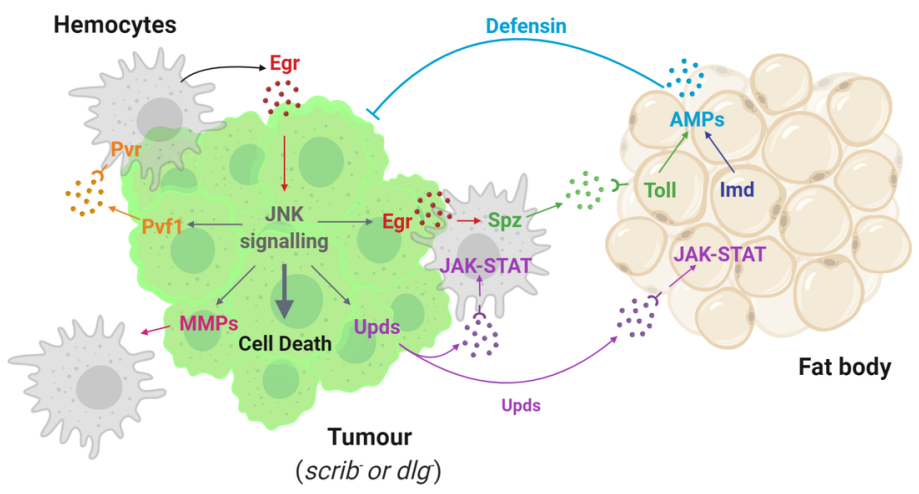

B

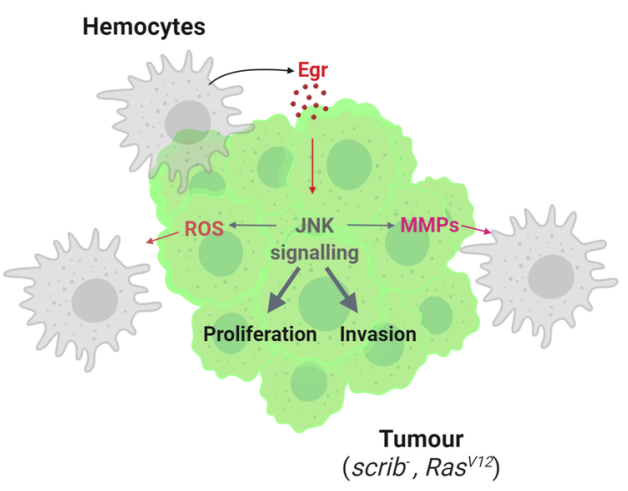

WDEV_394_Figure 2_v2.tif

This article is protected by copyright. All rights reserved. 


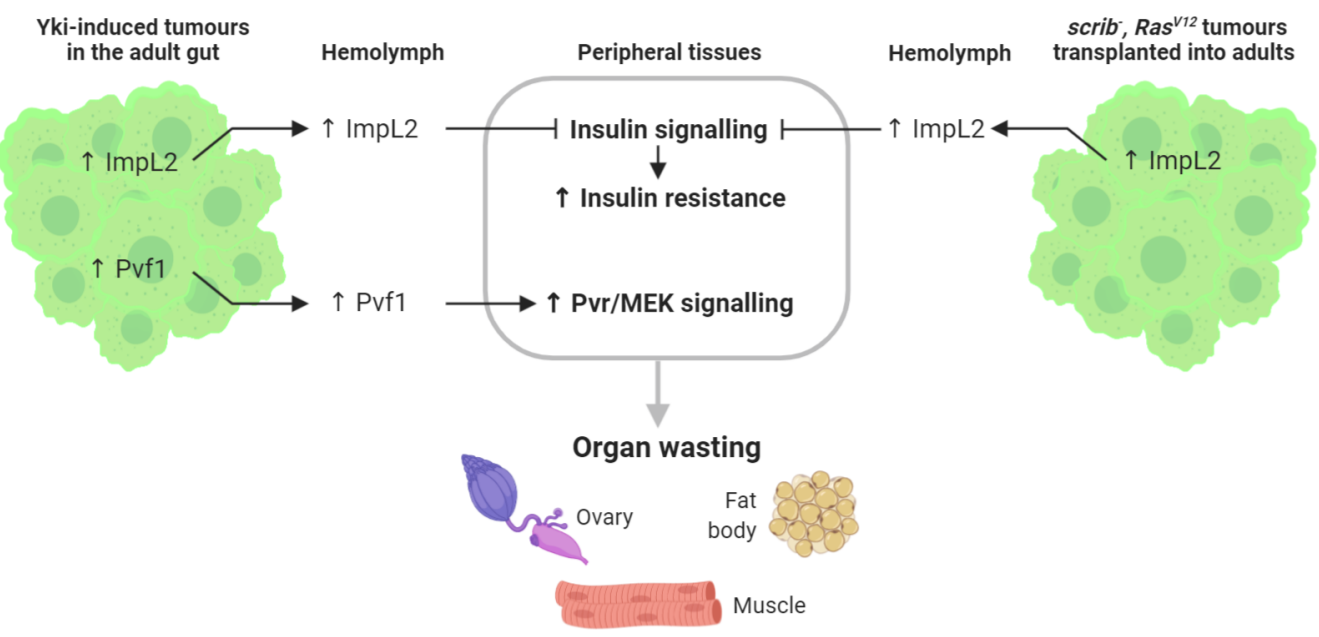

WDEV_394_Figure 3_v2.tif

This article is protected by copyright. All rights reserved. 


\section{University Library}

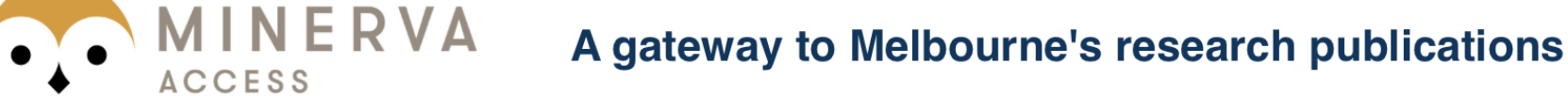

Minerva Access is the Institutional Repository of The University of Melbourne

Author/s:

Alvarez-Ochoa, E;Froldi, F;Cheng, LY

Title:

Interorgan communication in development and cancer

Date:

2020-08-27

Citation:

Alvarez-Ochoa, E., Froldi, F. \& Cheng, L. Y. (2020). Interorgan communication in development and cancer. WILEY INTERDISCIPLINARY REVIEWS-DEVELOPMENTAL BIOLOGY, 10 (4), https://doi.org/10.1002/wdev.394.

Persistent Link:

http://hdl.handle.net/11343/276192 